\title{
Gene expression in mouse ovarian follicle development in vivo versus an ex vivo alginate culture system
}

\author{
Elizabeth M Parrish ${ }^{1}$, Anaar Siletz ${ }^{1,7,8}$, Min Xu $\mathrm{u}^{2,5,7}$, Teresa K Woodruff ${ }^{2,3,5,6,7}$ and \\ Lonnie D Shea ${ }^{1,2,3,4,7}$
}

\begin{abstract}
${ }^{1}$ Department of Chemical and Biological Engineering, Northwestern University, 2145 Sheridan Road, Tech E136, Evanston, Illinois 60208, USA, ${ }^{2}$ Robert H Lurie Comprehensive Cancer Center, Northwestern University, Galter Pavilion, 675 N Saint Clair Street, 21st Floor, Chicago, Illinois 60611, USA, ${ }^{3}$ Chemistry of Life Processes Institute, Northwestern University, 2170 Campus Drive, Evanston, Illinois 60208-2850, USA, ${ }^{4}$ Institute for BioNanotechnology in Advanced Medicine, Northwestern University, 303 East Superior Street, Lurie Building, Chicago, Illinois 60611, USA, ${ }^{5}$ Department of Obstetrics and Gynecology, ${ }^{6}$ Department of Biochemistry, Molecular Biology and Cell Biology, ${ }^{7}$ Member of the Oncofertility Consortium and ${ }^{8}$ Medical Scientist Training Program, Feinberg School of Medicine, Northwestern University, Chicago, Illinois 60611, USA
\end{abstract}

Correspondence should be addressed to L D Shea at Department of Chemical and Biological Engineering, Northwestern University; Email: I-shea@northwestern.edu

E M Parrish and A Siletz contributed equally to this work

\begin{abstract}
Ovarian follicle maturation results from a complex interplay of endocrine, paracrine, and direct cell-cell interactions. This study compared the dynamic expression of key developmental genes during folliculogenesis in vivo and during in vitro culture in a 3D alginate hydrogel system. Candidate gene expression profiles were measured within mouse two-layered secondary follicles, multi-layered secondary follicles, and cumulus-oocyte complexes (COCs). The expression of 20 genes involved in endocrine communication, growth signaling, and oocyte development was investigated by real-time PCR. Gene product levels were compared between i) follicles of similar stage and ii) COCs derived either in vivo or by in vitro culture. For follicles cultured for 4 days, the expression pattern and the expression level of 12 genes were the same in vivo and in vitro. Some endocrine (cytochrome P450, family 19, subfamily A, polypeptide 1 (Cyp19a1) and inhibin $\beta A$ subunit (Inhba)) and growth-related genes (bone morphogenetic protein 15 (Bmp15), kit ligand (Kit), and transforming growth factor $\beta$ receptor 2 (Tgfbr2)) were downregulated relative to in vivo follicles. For COCs obtained from cultured follicles, endocrine-related genes (inhibin $\alpha$-subunit (Inha) and Inhba) had increased expression relative to in vivo counterparts, whereas growth-related genes (Bmp15, growth differentiation factor 9, and kit oncogene (Kit)) and zona pellucida genes were decreased. However, most of the oocyte-specific genes (e.g. factor in the germline $\alpha$ (Figla), jagged 1 (Jag1), and Nlrp5 (Mater)) were expressed in vitro at the same level and with the same pattern as in vivo-derived follicles. These studies establish the similarities and differences between in vivo and in vitro cultured follicles, guiding the creation of environments that maximize follicle development and oocyte quality.
\end{abstract}

Reproduction (2011) 142 309-318

\section{Introduction}

Ovarian follicle development is a highly complex process that requires coordination among the hypothalamus, the anterior pituitary, and the ovary, as well as bidirectional communication between the different cellular compartments within the follicle itself (Albertini et al. 2001, Erickson \& Shimasaki 2001, West et al. 2007a). Although many factors regulating this developmental process have been identified, the explicit role of these factors during early follicle development is enigmatic (Erickson \& Shimasaki 2000, Richards et al. 2002). Hormones that control follicle development can regulate expression of aromatase, inhibin, activin, FSH receptor, and LH receptor (McGee \& Hsueh 2000). Paracrine and autocrine signals, such as members of the transforming growth factor $\beta$ (TGFB) family, insulin-like growth factor (IGF), kit ligand, and anti-Mullerian hormone, derived from the follicular somatic cells and the oocyte, communicate bidirectionally between the cellular compartments and direct follicle development (Dong et al. 1996, Erickson \& Shimasaki 2000, Otsuka et al. 2000, Bristol \& Woodruff 2004, Juengel \& McNatty 2005, Knight \& Glister 2006). Finally, oocyte-specific gene products such as the zona pellucida proteins, factor in the germline alpha, and NLRP5 (MATER) have varying expression throughout development and impact follicle 
and oocyte maturation (Dean 2002). Working together, genes from these diverse categories form a communication network that results in the growth and development of the follicle and the oocyte, and most importantly the formation of a healthy and fertilizable egg.

Systems for in vitro ovarian follicle maturation have been developed and can be used to investigate the dynamics of gene expression throughout follicle development, which may aid in investigating the complex interplay of signals needed during follicle development (Kreeger et al. 2005, 2006, Xu et al. 2006a). The 3D culture system developed by our group consists of isolation of early mouse follicles followed by culture in alginate beads for the duration of follicle development to the antral stage. The alginate hydrogel allows growth in $3 \mathrm{D}$ and is thus referred to as a $3 \mathrm{D}$ culture system, in contrast to plating directly on a flat surface (2D). 2D culture of preantral mouse ovarian follicles on collagen membranes has resulted in live birth (Eppig \& Schroeder 1989, O'Brien et al. 2003). However, loss of the physical interactions between the oocyte and the granulosa cells that direct healthy follicle development is a concern in 2D (Cortvrindt et al. 1996). Supporting the natural 3D follicular structure may be more important for in vitro development of human ovarian follicles, which are larger than those of the mouse. The goal of our 3D model is to develop and characterize a culture system that can be successfully translated to human follicles for fertility preservation. Our system includes all of the cellular components necessary for follicular tissue development, as well as a chemical and mechanical environment that supports follicle growth and function in mouse ovarian follicles (West et al. 2007b). Importantly, the follicle does not require vascularization for development in vitro; therefore, this tissue can be cultured in isolation. The follicle maturation system supports follicle growth, steroid production, antrum formation, theca cell differentiation, and the development of mature fertilizable eggs that, following IVF and subsequent implantation into a host mother, have given rise to live, healthy, and fertile mouse pups (Xu et al. 2006a). Thus, this system enables a molecular-level investigation of in vitro follicle growth, which can be compared with freshly isolated follicles grown in vivo. This level of analysis provides insight into the similarities and differences in follicular development in the 3D alginate culture system versus the ovary.

In this study, we investigated the steady-state mRNA expression levels by real-time PCR of 20 developmental genes in ovarian follicles that were freshly isolated or cultured in vitro. The genes chosen for study are involved in endocrine signaling (cytochrome P450, family 19, subfamily A, polypeptide 1 (Cyp19a1), follicle-stimulating hormone receptor (Fshr), inhibin $\alpha$-subunit (Inha), inhibin $\beta$ A subunit (Inhba), and LH/choriogonadotropin receptor (Lhcgr)), follicular growth (insulin-like growth factor 1 (Igf1), kit oncogene (Kit), kit ligand (Kitl), and transforming growth factor $\beta$ receptor 2 (Tgfbr2)), and oocyte-specific functions (bone morphogenetic protein 15 (Bmp15), growth differentiation factor 9 (Gdf9), fibroblast growth factor 8 (Fgf8), factor in the germline $\alpha$ (Figla), jagged 1 (Jag1), NLR family, pyrin domain containing 5 (Nlrp5, also known as Mater), nobox oogenesis homeobox (Nobox), DEAD box polypeptide 4 $(D d \times 4$, also known as Vasa), zona pellucida glycoprotein $1(Z p 1), Z p 2$, and $Z p 3)$. This study provides a detailed analysis of the critical molecular events involved in the transition between follicle stages, which can be utilized to improve culture environments and thus maximize oocyte quality. In addition, this study quantifies the extent to which the culture system replicates physiology and tests the validity of the system for modeling follicular development.

\section{Results}

\section{In vivo and in vitro folliculogenesis}

The objective of the studies was to investigate the expression of genes associated with endocrine communication, paracrine signaling, and oocyte development during follicle growth. For in vivo studies, expression was analyzed for two-layered secondary (2LS) follicles, multi-layered secondary (MLS) follicles, and cumulus-oocyte complexes (COCs; Fig. 1A).

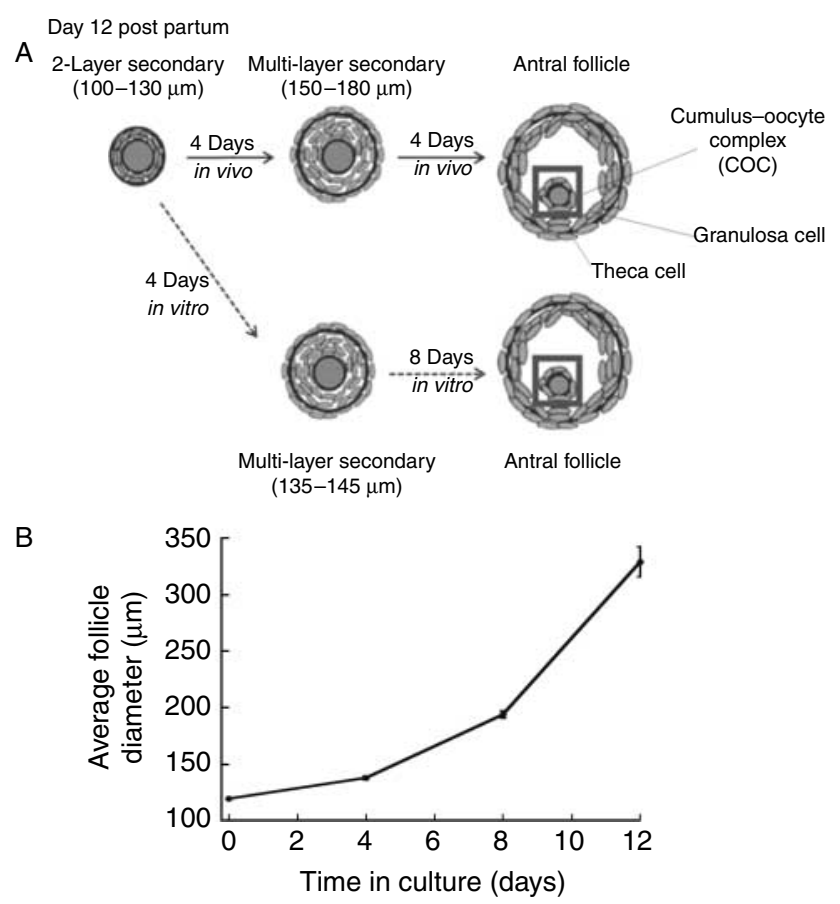

Figure 1 Two-layered secondary follicle growth in the $0.25 \%$ alginate culture system. Schematic illustrating in vivo follicular development versus development in vitro in $0.25 \%$ alginate beads, starting from $2 \mathrm{LS}$ follicles from $12 \mathrm{dpp}$ mice (A). Freshly isolated follicles had an average diameter of $119.1 \pm 1.14 \mu \mathrm{m}$ and after 12 days of culture grew to $329.07 \pm 13.38 \mu \mathrm{m}$ in diameter (B). 
2LS follicles, which have two layers of granulosa cells surrounding the oocyte and a diameter ranging from 100 to $130 \mu \mathrm{m}$, were isolated from day 12 mouse ovaries. MLS follicles were isolated from day 16 mouse ovaries and have multiple layers of granulosa cells surrounding the oocyte and a diameter ranging from 150 to $180 \mu \mathrm{m}$. For the in vitro cultures, gene expression was analyzed in follicles that had been cultured within $0.25 \%$ alginate hydrogels for 4 days, which have similar diameter and morphology to MLS follicles isolated in vivo from the day 16 ovary (Fig. 1A). In addition, gene expression was analyzed in COCs isolated from follicles that had been cultured for 12 days in $0.25 \%$ alginate.

During the 12-day culture period, the follicles increased in size and developed antral cavities. The alginate culture system maintained the 3D structure of the follicles (Kreeger et al. 2006), and follicles increased in size from an initial diameter of $119.1 \pm 1.1$ to 329.1 $\pm 13.4 \mu \mathrm{m}$ after 12 days of culture (Fig. 1B). The size of antral follicles achieved in vitro was smaller than antral follicle diameter in vivo, which is around $424 \mu \mathrm{m}$ (Griffin et al. 2006). Antrum formation was also observed in this culture system, with antra developing after 8-10 days of culture (Fig. 1A). This growth curve is consistent with the follicle growth and development that has previously been described (Xu et al. 2006b).

\section{Gene expression analysis}

To compare follicle development in vitro and in vivo, gene expression in vivo in MLS follicles isolated from the day 16 ovary was compared to in vitro follicles isolated from the day 12 ovary that had been cultured for 4 days (Fig. 1A). In addition, gene expression from in vivo COCs was compared to expression within COCs obtained from follicles after 12 days of culture.

\section{Endocrine-related genes}

The expression of five genes involved in endocrine communication was quantified during in vivo and in vitro development of ovarian follicles. Steady-state mRNA encoding aromatase (Cyp19a1), the FSH receptor (Fshr), Inha, Inhba, and Lhcgr were assessed. In vivo, all endocrine-related genes, with the exception of Lhcgr, increased in expression from $2 \mathrm{LS}$ to MLS with a fold change of at least 2 (Fig. 2A). Lhcgr did not exhibit a significant change in expression. In vitro, three of the five endocrine genes had similar patterns of expression as the in vivo isolated follicles. Fshr was expressed in both in vivo and in vitro MLS follicles with at least two times the expression level of 2LS follicles and Inha expression was two times greater in all MLS follicles than in 2LS follicles (Fig. 2A and B). Lhcgr expression levels did not change during in vitro culture or in the in vivo condition. However, expression levels of Inhba did not change
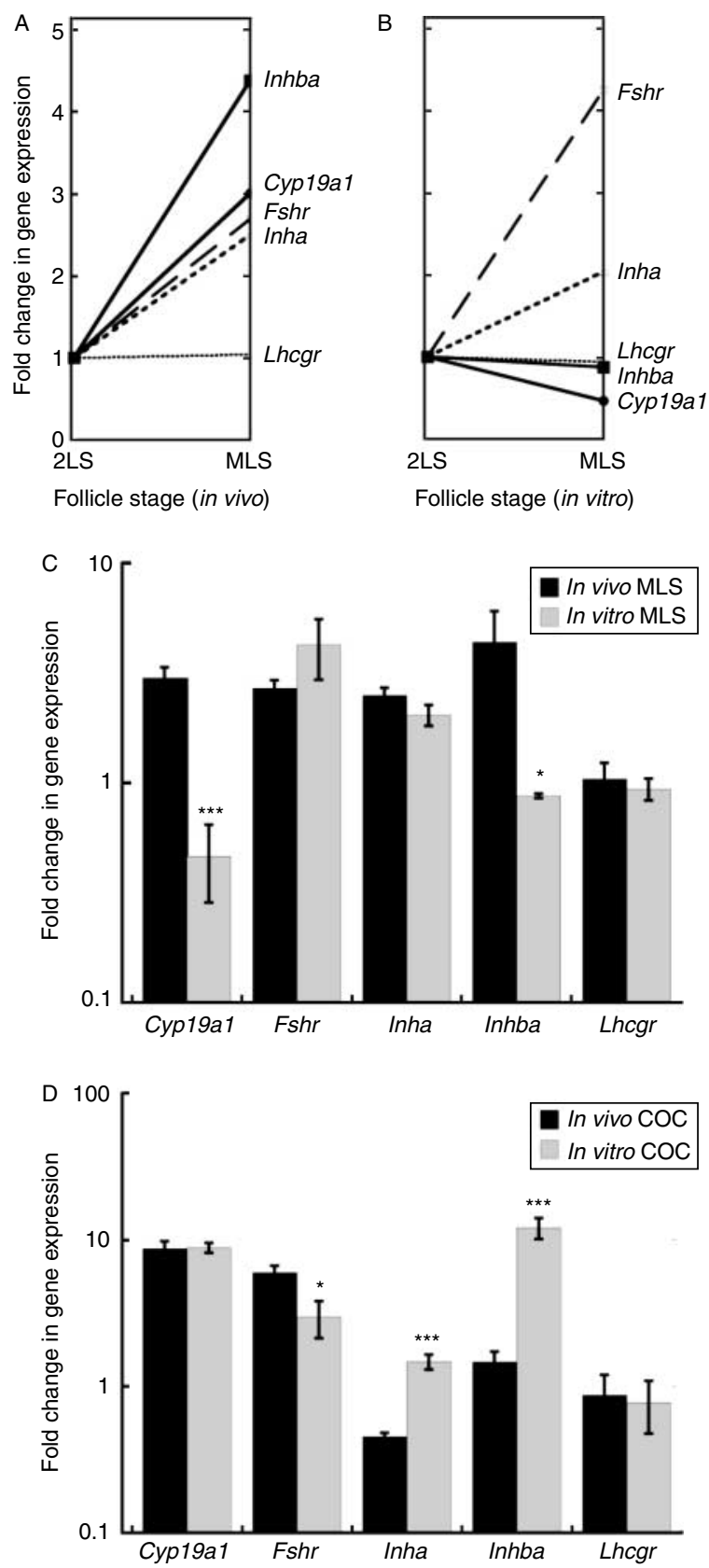

Figure 2 Fold changes in gene expression as measured by real-time PCR for genes involved in endocrine signaling. Changes in gene expression are shown as follicles develop from $2 \mathrm{LS}$ follicles isolated from $12 \mathrm{dpp}$ ovaries to MLS follicles isolated from $16 \mathrm{dpp}$ ovaries (in vivo developed) (A) or from $2 \mathrm{LS}$ follicles isolated from $12 \mathrm{dpp}$ follicles and cultured to the MLS stage over 4 days (in vitro developed) (B). Note Lhcgr and Inhba overlie each other in (B). Fold changes in gene expression relative to $2 \mathrm{LS}$ follicles are directly compared between in vivo MLS follicles (black bars) and follicles cultured for 4 days in vitro (gray bars) (C) and between in vivo COCs from antral follicles (black bars) and COCs from follicles cultured for 12 days in vitro (gray bars) (D). Error bars represent the S.E.M. ${ }^{*} P<0.05$ and ${ }^{* * *} P<0.001$. Note log scale for (C) and (D). $N=3$ for all experiments. 
between 2LS and MLS in vitro, in contrast with the in vivo results (Fig. 2C). Also in contrast with in vivo results, Cyp19a1 levels declined between 2LS and MLS follicles in vitro (Fig. 2B). Cyp19a1 and Inhba had significantly (6.5-and 5-fold) reduced expression levels within the MLS follicles in vitro relative to in vivo. Thus, Fshr, Inha, and Lhcgr had the same pattern of expression in vivo as in vitro, but Cyp19a1 and Inhba differed between the in vivo and in vitro conditions.

For COCs, the expression levels varied between the in vitro and the in vivo conditions for three of the five factors. Cyp19a1 and Lhcgr had similar expression levels in vitro as in vivo (Fig. 2D). Inha and Inhba were upregulated in in vitro COCs compared to in vivo, which contrasts with the MLS studies that indicated no change or downregulation for in vitro relative to in vivo follicles. Fshr had twofold reduced expression in vitro compared with in vivo $(P<0.05)$.

\section{Growth-related genes}

We subsequently investigated the expression patterns of four genes involved in growth signaling: Igf1, Tgfbr2, Kit, and Kitl (Fig. 3). In vivo, Igf1 levels substantially increased (4.5-fold) between the 2 LS and the MLS stages whereas in vitro Igf1 levels increased 1.8-fold. Tgfbr2 and Kit levels in vivo increased to a lesser extent (1.4and 1.8-fold respectively), and expression levels of Kitl did not significantly change. Kit had a similar expression pattern in vitro as in vivo, with levels slightly increased during development to the MLS stage. Kitl and Tgfbr2 had decreased expression after 4 days of culture (2-fold and 4.5-fold respectively, relative to 2 LS follicles), which contrasts with the in vivo results (Fig. $3 \mathrm{~B}$ ). Relative to in vivo follicles, Kitl and Tgfbr2 were downregulated in cultured MLS follicles 6-fold and 2.4-fold respectively (Fig. 3C). Igf1 was the only non-oocyte-specific growthrelated gene to be expressed at least twofold higher in MLS follicles compared with $2 \mathrm{LS}$ follicles in vivo. For COCs, only one of the four genes had a significantly different expression level between cultured and freshly isolated follicles. Kit expression differed between in vitro and in vivo conditions, whereas Igf1, Kitl, and Tgfbr2 were expressed similarly between in vivo and in vitro COCs (Fig. 3D).

\section{Oocyte-specific genes}

The expression levels of 11 genes that, in the ovarian follicle, are specifically expressed in the oocyte were measured. The designation 'oocyte-specific' is used to indicate that these factors are expressed within the oocyte and does not capture the multi-functional roles of these genes in follicle development and oocyte maturation. In vivo, levels of Bmp15, Nobox, Ddx4, and Zp2 increased by factors of 1.7-, 1.7-, 2.6-, and 1.9-fold from

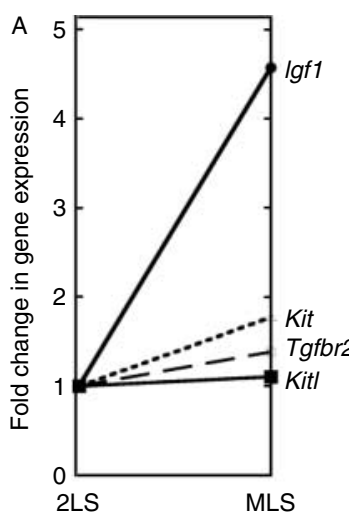

Follicle stage (in vivo)
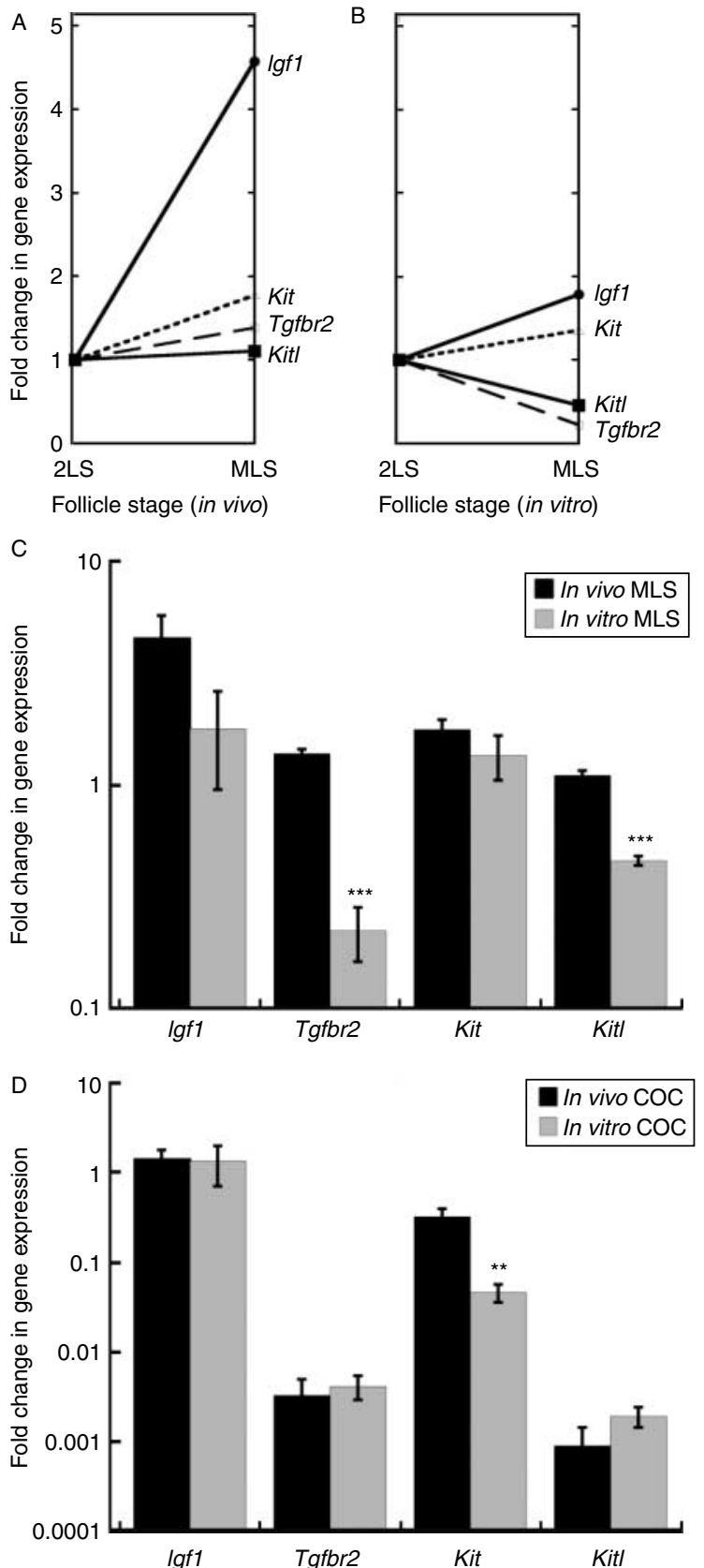

Figure 3 Fold changes in gene expression as measured by real-time PCR for genes involved in growth signaling. Changes in gene expression are shown as follicles develop from $2 \mathrm{LS}$ follicles isolated from $12 \mathrm{dpp}$ ovaries to MLS follicles isolated from $16 \mathrm{dpp}$ ovaries (in vivo developed) (A) or from 2 LS follicles isolated from $12 \mathrm{dpp}$ follicles and cultured to the MLS stage over 4 days (in vitro developed) (B). Fold changes in gene expression relative to 2 LS follicles are directly compared between in vivo MLS follicles (black bars) and follicles cultured for 4 days in vitro (gray bars) (C) and between in vivo COCs from antral follicles (black bars) and COCs from follicles cultured for 12 days in vitro (gray bars) (D). Error bars represent the S.E.M. ${ }^{* *} P<0.01$ and $* * * P<0.001$. Note log scale for (C) and (D). $N=3$ for all experiments. 

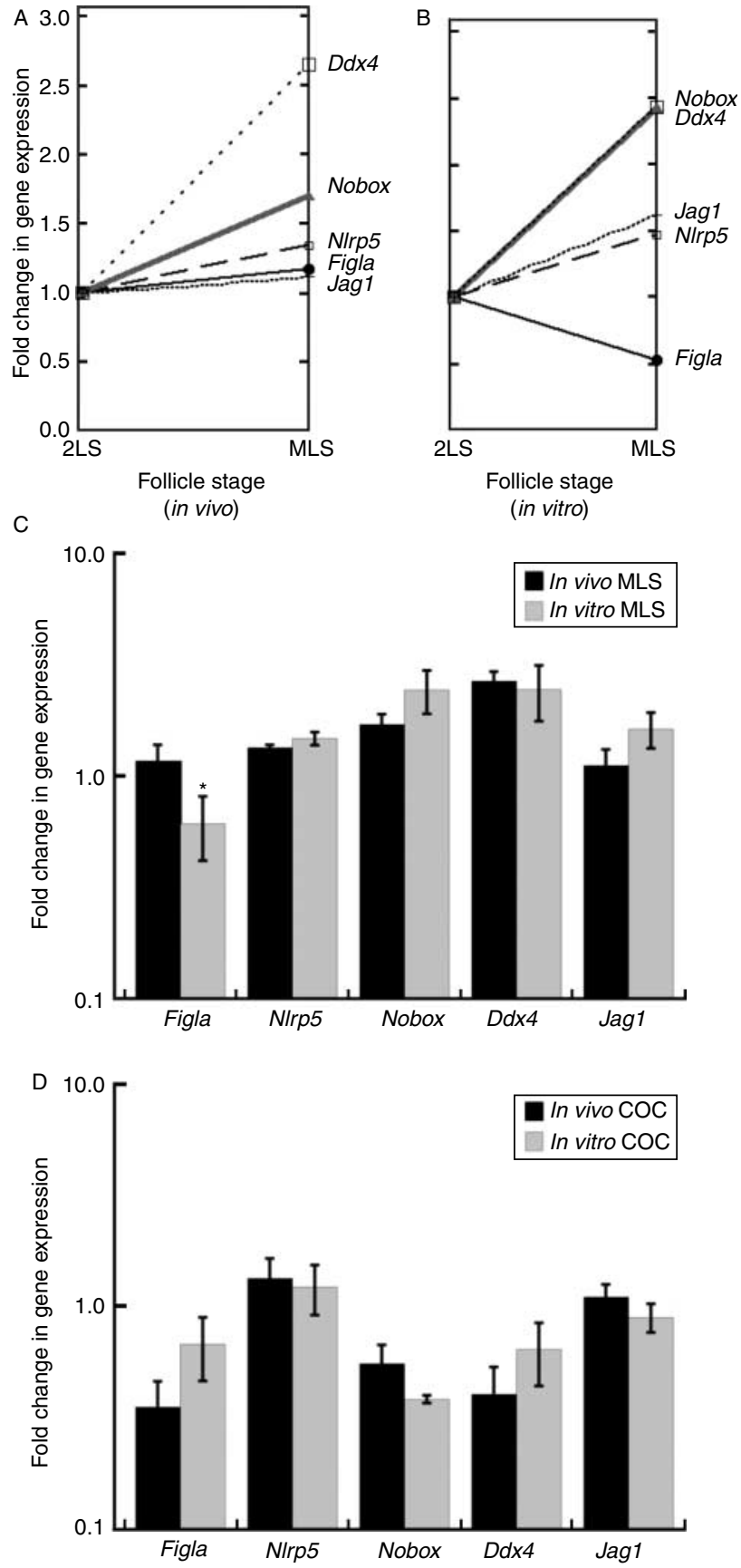

Figure 4 Fold changes in gene expression as measured by real-time PCR for genes involved in oocyte development that are expressed in vitro at the same level as in vivo. Changes in gene expression are shown as follicles develop from 2 LS follicles isolated from $12 \mathrm{dpp}$ ovaries to MLS follicles isolated from 16 dpp ovaries (in vivo-developed) (A) or from $2 \mathrm{LS}$ follicles isolated from $12 \mathrm{dpp}$ follicles and cultured to the MLS stage over 4 days (in vitro developed) (B). Note Nobox and $D d x 4$ overlie each other in (B). Fold changes in gene expression relative to 2 LS follicles are directly compared between in vivo MLS follicles (black bars) and follicles cultured for 4 days in vitro (gray bars) (C) and between in vivo COCs from antral follicles (black bars) and COCs from follicles cultured for 12 days in vitro (gray bars) (D). Although Figla was significantly downregulated at the MLS stage in vitro, there was no significant difference in vitroversus in vivo by the COC stage. Error bars represent the S.E.M. Note log scale for (C) and (D). ${ }^{*} P<0.05 . N=3$ for all experiments. the $2 \mathrm{LS}$ to MLS stage (Fig. $4 \mathrm{~A}$ and C; Fig. $5 \mathrm{~A}$ and C). Levels of Figla, Gdf9, Jag1, Nlrp5, Zp1, and Zp3 varied by $33 \%$ or less between 2 LS and MLS. Fgfo levels were significantly downregulated in MLS follicles compared to $2 \mathrm{LS}$ follicles. For in vitro culture to the MLS stage, the expression of Nobox, $D d x 4$, and $Z p 2$ had significantly increased expression between $2 \mathrm{LS}$ and MLS, with Nobox expression having a greater increase in vitro than in vivo (Fig. 4A and B; Fig. 5B). The expression of NIrp5, Jag1, Bmp15, Zp1, and Zp3 changed little over the 4-day culture period (Fig. 4B; Fig. 5B). Expression of NIrp5 and Jag1 also changed minimally in vivo over the same time period. However, in vitro levels of $B m p 15, Z p 1$, and $Z p 3$ were significantly different between in vivo and in vitro MLS follicles (Fig. 5C). Figla, Fgf8, and Gdf9 had decreasing levels of expression between the 2LS and the MLS stages in vitro, which was only observed with Fgf8 in vivo. Of the 11 genes, four had significantly different transcript levels between MLS follicles in vitro and in vivo: Bmp15, Figla, Zp1, and Zp3. Zp3 had an increased level of expression in vitro relative to in vivo, whereas the remainder had decreased expression levels in vitro.

The expression of Bmp15, Fgf8, Gdf9, Zp1, Zp2, and $Z p 3$ was significantly different for in vivo and in vitroderived COCs. Fgfo had increased expression levels in vitro relative to in vivo, whereas the other five factors had expression that was reduced in vitro relative to in vivo (Fig. 5D). The expression levels of Figla, Nlrp5, Nobox, $D d x 4$, and Jag1 were not significantly different between in vivo COCs and COCs obtained from antral follicles at the end of culture (Fig. 4D).

\section{Discussion}

This study investigated expression patterns of genes known to be important in folliculogenesis during in vivo follicle development and during in vitro culture. The expression levels of 20 genes involved in follicle development were quantified in 2LS, MLS, and COCs derived from antral follicles. Table 1 shows the gene regulation patterns, categorized as increased, decreased, or unchanged relative to $2 \mathrm{LS}$ follicles, for MLS follicles in vivo. For the endocrine-related genes in in vivo isolated follicles, Cyp19a1 expression increased from the 2LS to the MLS state, which is consistent with induction within the granulosa cells of preovulatory follicles (Hickey et al. 1988). Fshr is solely expressed in granulosa cells of developing follicles, with twofold increased expression in preovulatory follicles (LaPolt et al. 1992, Sites et al. 1994) as is consistent with this report. In addition, the expression of both inhibins and activins has been reported to be maximal in ovaries in which secondary follicles dominate (Woodruff et al. 1988, Findlay et al. 2001, Pangas et al. 2002), which is also consistent with our mRNA data. Finally, $\mathrm{LH}$ receptor expression is induced in mural cells of preovulatory 

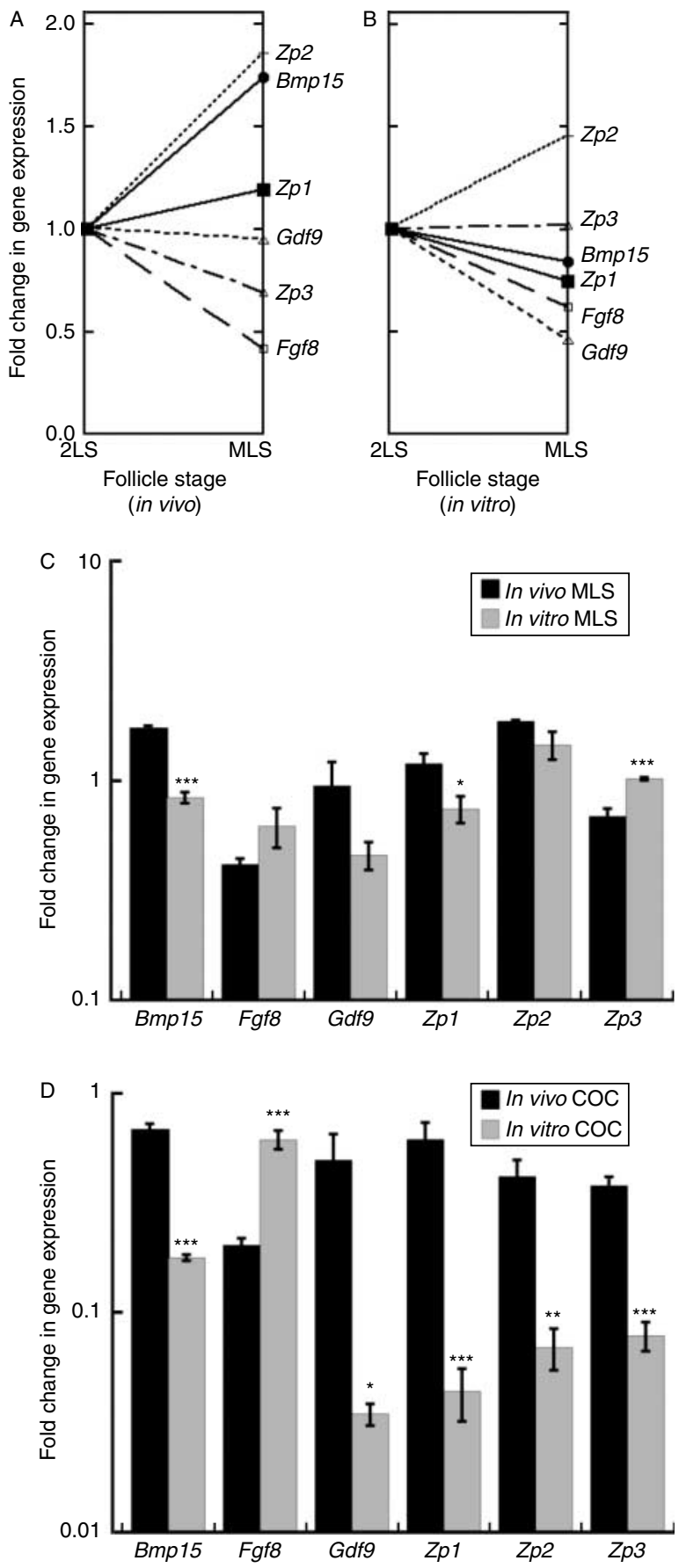

Figure 5 Fold changes in gene expression as measured by real-time PCR for genes involved in oocyte development that are expressed differentially between in vivo and in vitro conditions. Changes in gene expression are shown as follicles develop from $2 \mathrm{LS}$ follicles isolated from $12 \mathrm{dpp}$ ovaries to MLS follicles isolated from $16 \mathrm{dpp}$ ovaries (in vivo developed) (A) or from $2 \mathrm{LS}$ follicles isolated from $12 \mathrm{dpp}$ follicles and cultured to the MLS stage over 4 days (in vitro developed) (B). Fold changes in gene expression relative to $2 \mathrm{LS}$ follicles are directly compared between in vivo MLS follicles (black bars) and follicles cultured for 4 days in vitro (gray bars) (C) and between in vivo COCs from antral follicles (black bars) and COCs from follicles cultured for 12 days in vitro (gray bars) (D). Error bars represent the S.E.M. ${ }^{*} P<0.05,{ }^{* *} P<0.01$, and ${ }^{* * *} P<0.001$. Note log scale for (C) and (D). $N=3$ for all experiments. follicles. For the transition from $2 \mathrm{LS}$ to MLS, Lhcgr levels were unchanged, which was expected given the early stage of follicle development (Segaloff et al. 1990, Lei et al. 2001).

Substantial growth of the follicle and development of the oocyte occur during development from the 2LS state to ultimately produce a COC. Thus, we investigated the expression of multiple growth factor, as well as oocytespecific transcripts. A significant increase in expression of Igf1 at the MLS follicle stage was observed (Table 1), consistent with its role in oocyte maturation (McGee \& Hsueh 2000). For the in vivo isolated follicles, the expression of many growth-related genes reported here (Bmp15, Gdf9, Kit, Kitl, and Tgfbr2) changed less than twofold from the 2LS to the MLS follicle stage (Table 1), consistent with the roles of these genes in the regulation of cell fate decisions during the preantral phase of folliculogenesis (Parrott \& Skinner 1997, Driancourt et al. 2000, Knight \& Glister 2006, Thomas \& Vanderhyden 2006). Patterns of oocyte-specific gene expression observed within the in vivo isolated follicles (Table 1) are also supported by previous reports of gene function (Suzumori et al. 2002, Shimasaki et al. 2004, Knight \& Glister 2006). NIrp5 and Jag1 are the only two genes that have steady or increased expression at the COC stage compared to the 2LS follicle stage (Fig. 4C), consistent with their roles in oocyte growth. NIrp5 expression reportedly persists into the blastocyst stage of embryonic development (Dean 2002), and Jag1 is an important factor during all stages of folliculogenesis (Vorontchikhina et al. 2005). The expression patterns of Figla and the genes encoding the zona pellucida proteins in vivo (Table 1, Fig. $4 \mathrm{~A}$ and $\mathrm{C}$, Fig. $5 \mathrm{~A}$ and $\mathrm{C}$ ) are also consistent with previous reports that Figla regulates and is required for zona pellucida gene expression and that this expression increases and then decreases as the oocyte increases in diameter (Epifano et al. 1995).

In this study, we investigated gene expression that occurs during in vivo and in vitro follicle growth as a means to identify the supportive and limiting components of the alginate culture environment. In comparing the patterns of expression, we determined that $60 \%$ of in vitro candidate gene patterns investigated are similar to their corresponding in vivo patterns of expression at the MLS stage (Table 2), whereas 50\% of the factors had similar expression patterns in COCs in vitro and in vivo (Table 3). As the culture system supports follicle growth, antrum formation, steroid production, and ultimately the production of fertilizable oocytes, it is expected that many of the gene expression patterns in our culture system are the same as those in vivo. At the MLS stage, Fgf8, Fshr, Gdf9, Igf1, Inha, Jag1, Kit, Lhcgr, Nlrp5, Nobox, Ddx4, and Zp2 were expressed at similar levels in vivo and in vitro. Of these, expression levels of Fgf8, Fshr, Gdf9, Kit, and Zp2 had diverged by the COC stage (Table 3). However, Cyp19a1, Figla, Kitl, and Tgfbr2 levels were not 
Table 1 Patterns of gene expression as follicles develop in vivo from the two-layered secondary to multi-layered secondary stages. Expression changes of twofold or greater at the multi-layered secondary follicle stage are considered increased or decreased from the twolayered secondary follicle stage, whereas changes of less than twofold are reported here as unchanged.

\begin{tabular}{|c|c|c|c|}
\hline & Increase & Decrease & No change \\
\hline \multicolumn{4}{|l|}{ In vivo } \\
\hline Endocrine & $\begin{array}{l}\text { Cyp19a1 } \\
\text { Fshr } \\
\text { Inha } \\
\text { Inhba }\end{array}$ & & Lhcgr \\
\hline \multirow[t]{5}{*}{ Growth } & $\operatorname{lgf1}$ & & Bmp15 \\
\hline & & & Gdf9 \\
\hline & & & Kit \\
\hline & & & Kitl \\
\hline & & & Tgfbr2 \\
\hline \multirow[t]{7}{*}{ Oocyte } & $D d x 4$ & Fgf8 & Figla \\
\hline & & & Jag1 \\
\hline & & & Nlrp5 \\
\hline & & & Nobox \\
\hline & & & Zp1 \\
\hline & & & Zp2 \\
\hline & & & Zp3 \\
\hline
\end{tabular}

significantly different between in vivo and in vitro COCs. The oocyte has been described as orchestrating folliculogenesis as well as its own maturation, and the expression of these genes in similar patterns and at comparable levels between in vitro and in vivo is consistent with the generation of healthy, viable, and meiotically competent oocytes obtained from our culture system (Dean 2002, Eppig et al. 2002, Xu et al. 2006a, 2006b).

While in vitro culture does promote follicle growth and oocyte development, the follicles cultured in vitro do not reach the same final diameter as in vivo grown follicles and the oocytes do not have the same potential for blastocyst development and production of live births. These observations motivated this study to identify differences between in vitro cultured follicles and in vivo follicles, which were observed primarily as quantitative differences in expression level and not a complete absence of expression. Differences were expected due to isolating follicles from their native environment, which may alter the signals that normally regulate follicle development. For example, signaling through the hypothalamic-pituitary-gonadal axis is disrupted, surrounding follicles and somatic cells are removed and oxygen concentrations differ. While only $15 \%$ of the tested genes were more highly expressed in in vitro COCs compared with in vivo COCs, $35 \%$ of the tested genes had lower expression levels in vitro compared with in vivo (Table 3). Of the endocrinerelated genes, only Lhcgr was expressed in the same manner and at the same level of expression between in vivo and in vitro cultured follicles and COCs. Inha and Inhba were upregulated in the cultured COCs compared to in vivo COCs, which suggest some disruption of normal endocrine regulation of development. In addition, increased inhibin levels may be required for in vitro follicle development, in order to accommodate the decrease in the external stimuli that normally regulate development.

Substantial differences in transcript levels of oocytespecific growth factor and zona pellucida genes were observed (Table 3), as well as a difference in overall expression patterns of Fgf8, Kitl, Tgfbr2, and Zp3 (Table 2). Decreased expression of the growth-related genes, such as Bmp15, Gdf9, and Kit, may be a consequence of removing the follicle from growth inhibitory signals imparted by surrounding follicles and stromal cells. In the absence of this inhibition, the reduced expression of these genes may be sufficient to promote follicle development. However, these variations in expression level between in vitro and in vivo may also underlie the differences in the follicle and oocyte properties that are obtained at the conclusion of culture.

This culture system provides an enabling tool to investigate the differences in order to understand the contributions of the environment to follicle development. Importantly, we note that the in vitro and in vivo environment has significant differences from each other, as well as the normal cycle. Pregnant mare serum gonadotropin (PMSG) raises the level of $\mathrm{FSH}$ above normal in order to induce the development of preovulatory follicles. Although this technique is effective, it is not reflective of the normal cycle. For our in vitro culture, FSH levels are constant, which is also not reflective of the normal cycling mouse. Although these approaches to obtain mature oocytes differ and may underlie some of the differences in gene expression, a number of similarities are present in the measurements of gene expression.

Table 2 Patterns of gene expression as follicles develop in vitro from the two-layered secondary to multi-layered secondary stages. Bolded genes indicate significant differences relative to the in vivo condition.

Expression changes of twofold or greater at the multi-layered secondary follicle stage are considered increased or decreased from the two-

layered secondary follicle stage, whereas changes of less than twofold are reported here as unchanged.

\begin{tabular}{llll}
\hline & Increase & Decrease & No change \\
\hline $\begin{array}{lll}\text { In vitro } \\
\text { Endocrine }\end{array}$ & Fshr & Cyp19a1 & Inhba \\
& Inha & Kitl & Lhcgr \\
Growth & & Tgfbr2 & Bmp15 \\
& & Fgf8 \\
& & Gdf9 \\
& & Kit \\
& & Igf1 \\
& & Figla \\
Oocyte & Nobox & & Jag1 \\
& Ddx4 & & Nlrp5 \\
& & Zp1 \\
& & Zp2 \\
& & Zp3 \\
\hline
\end{tabular}


Table 3 Summary of mRNAs that are higher or lower under in vitro versus in vivo conditions at the multi-layered secondary follicle (MLS) stage or the cumulus-oocyte complex (COC) stage.

\begin{tabular}{|c|c|c|c|c|}
\hline & \multicolumn{2}{|c|}{ MLS follicles } & \multicolumn{2}{|c|}{ COCs } \\
\hline & Up in vitro & Down in vitro & Up in vitro & Down in vitro \\
\hline Endocrine & & $\begin{array}{l}\text { Cyp19a1 } \\
\text { Inhba }\end{array}$ & $\begin{array}{l}\text { Inha } \\
\text { Inhba }\end{array}$ & Fshr \\
\hline Growth & & $\begin{array}{l}\text { Bmp15 } \\
\text { Kitl } \\
\text { Tgfbr2 }\end{array}$ & & $\begin{array}{l}\text { Bmp15 } \\
\text { Gdf9 } \\
\text { Kit }\end{array}$ \\
\hline Oocyte & $Z p 3$ & $\begin{array}{l}\text { Figla } \\
\text { Zp1 }\end{array}$ & Fgf8 & $\begin{array}{l}Z p 1 \\
Z p 2 \\
Z p 3\end{array}$ \\
\hline
\end{tabular}

In this study, the 3D alginate culture system was shown to support follicular gene expression similar, in part, to that of follicles grown in vivo. These similarities between in vitro and in vivo validate the use of the 3D culture system as a convergent tool for investigating ovarian follicular development and modeling follicle biology. The results of this study suggest that the 3D alginate culture system could be a powerful tool for modeling other physiologic processes, which depend on a 3D architecture and which are affected by environmental rigidity. For example, culture in $2 \mathrm{D}$ versus $3 \mathrm{D}$ alters the differentiation potential of embryonic stem cells (Dawson et al. 2008) and matrix rigidity plays a role in the differentiation potential of stem cells and cancer progression. A 3D alginate culture system of varying rigidity, combined with appropriate growth factors, can be used as a synthetic environment to molecularly dissect tissue formation.

There were also many differences in gene expression between follicles grown in the alginate culture system and those grown in vivo. Because follicles in culture are removed from the context of endocrine axes and the paracrine effects of neighboring follicles, gene expression patterns supporting optimized follicle growth may have differed from those in vivo. Thus, it cannot be assumed that all differences seen between in vitro and in vivo follicles are maladaptive. Nevertheless, genes crucial for healthy follicle development, which are expressed differently in the alginate culture system, are potential targets of, and markers for, improved culture conditions that support a higher frequency of fertilizable oocyte development.

In conclusion, the abundance and changing transcription patterns of gene expression during both in vivo and in vitro folliculogenesis were directly compared within MLS follicles and COCs. For the genes tested, $60 \%$ had the same in vitro patterns of gene expression during the first 4 days of culture as follicles isolated in vivo with a similar size and morphology. In addition, half of the factors had the same expression level within COCs obtained in vivo and following in vitro culture. Interestingly, oocyte-specific genes such as Figla, Jag1, Nlrp5, Nobox, and Ddx4 were all expressed in vitro at the same level and with the same pattern as they were in vivo. Despite the many similarities, several differences in expression profile were noted. Endocrine-related genes, such as Cyp19a1 and Inhba, as well as growthrelated genes, such as Bmp15, Kitl, and Tgfbr2, had lower levels of expression in vitro than in vivo after 4 days of culture. At the culmination of culture, COCs obtained in vitro expressed endocrine-related genes (Fshr, Inha, and Inhba), growth-related genes (Kit and the oocyte-specific genes Bmp15 and Gdf9), and other oocyte-specific genes (Fgf8, Zp1, Zp2, and Zp3) at aberrant levels compared to in vivo COCs. These studies provide a set of gene expression profiles indicating the similarities and differences between in vivo and in vitro cultured follicles, providing a basis for investigating follicular development and the creation of environments that promote follicle development and maximize oocyte quality.

\section{Materials and Methods}

\section{Animals and materials}

Male $C B A$ and female $C 57 B L / 6$ mouse breeding pairs were housed in a temperature- and light-controlled environment (12 h light:12 h darkness) and provided with food and water ad libitum. Animals were fed Teklad Global irradiated 2919 chow, free of phyto-estrogens. Animals were treated in accordance with the National Institutes of Health Guide for the Care and Use of Laboratory Animals and protocols approved by the Institutional Animal Care and Use Committee at Northwestern University.

\section{Alginate preparation}

Sodium alginate (55-65\% glucuronic acid) was provided by FMC BioPolymers (Philadelphia, PA, USA). Charcoal-stripped and sterilized alginate was reconstituted with $1 \times \mathrm{PBS}$ to a concentration of $0.25 \%(\mathrm{w} / \mathrm{v})$.

\section{Follicle isolation, encapsulation, and culture}

2LS (follicular diameter of 100-130 $\mu \mathrm{m}$ ) and MLS (follicular diameter of 150-180 $\mu \mathrm{m}$ ) were mechanically isolated from ovaries of C57BL/6xCBA F1 mice 12 and 16 days postpartum (dpp) respectively (Xu et al. 2006a). 2 LS and MLS follicles were immediately frozen in clean tubes with minimal media for realtime PCR analysis of in vivo gene expression. For analysis of gene expression in the alginate culture system, 2LS follicles were encapsulated into $0.25 \%(\mathrm{w} / \mathrm{v})$ alginate beads for culture, as described previously (Xu et al. 2006 b). Growth medium was prepared using $\alpha-M E M$ with $10 \mathrm{mIU} / \mathrm{ml}$ recombinant $\mathrm{FSH}$ (Organon, Roseland, NJ, USA), $3 \mathrm{mg} / \mathrm{ml}$ BSA, $1 \mathrm{mg} / \mathrm{ml}$ bovine fetuin (Sigma-Aldrich), $5 \mu \mathrm{g} / \mathrm{ml}$ insulin, $5 \mu \mathrm{g} / \mathrm{ml}$ transferrin, and $5 \mathrm{ng} / \mathrm{ml}$ selenium. Beads containing follicles were cultured at $37{ }^{\circ} \mathrm{C}$ and $5 \% \mathrm{CO}_{2}$ in individual wells of a 96-well plate containing $100 \mu \mathrm{l}$ growth medium for 4-12 days, with half of the medium replaced with fresh medium every second day. 
Images of the follicles were taken every 4 days using a Leica DM IL light microscope (Leica, Wetzlar, Germany) equipped with a heated stage, and a Spot Insight 2 Megapizel Color Mosaic camera with Spot software (Spot Diagnostic Instruments, Sterling Heights, MI, USA). Follicle diameters were measured using ImageJ Software (National Institutes of Health, Bethesda, MD, USA). After the culture period, the medium was replaced with $100 \mu \mathrm{L}$ L15 medium containing $10 \mathrm{IU} / \mathrm{ml}$ alginate lyase (Sigma-Aldrich) for $30 \mathrm{~min}$ at $37^{\circ} \mathrm{C}$ and $5 \% \mathrm{CO}_{2}$ to degrade the alginate bead and release the follicle into the medium. For each RNA sample, an average of 30 follicles were removed from medium and immediately frozen in clean tubes in minimal medium for RNA isolation. For each time point, three independent samples were collected. Each sample consisted of follicles isolated from multiple mice from the same litter. Separate samples, either for in vivo analysis or for in vitro culture, were isolated on different days from different litters.

\section{COC isolation}

For in vivo $\mathrm{COC}$ isolation, $C 57 \mathrm{BL} / 6 \times \mathrm{CBA} \mathrm{F} 1$ mice $18 \mathrm{dpp}$ were i.p. injected with $5 \mathrm{IU}$ PMSG. Ovaries were collected $40 \mathrm{~h}$ post-injection, and COCs were mechanically isolated from antral follicles of the stimulated ovaries. COCs were also isolated from antral follicles derived from culturing $2 \mathrm{LS}$ follicles from 12 dpp mice for 12 days, as described above; thus, these antral follicles were $24 \mathrm{dpp}$ at the time of isolation. Approximately 15-20 antral follicles for RNA isolation were punctured to release the COC for each sample. COCs were pipetted into clean tubes and immediately frozen in minimal media for RNA isolation. In total, three independent samples were collected as for $2 \mathrm{LS}$ and MLS follicles above.

\section{Real-time PCR}

Total RNA from frozen uncultured or cultured follicles was isolated using the Absolutely RNA Microprep Kit (Stratagene, Cedar Creek, TX, USA), following the manufacturer's instructions. RNA was reverse transcribed into first-strand cDNA using the SuperScript First Strand Synthesis System (Invitrogen) with random hexamer primers. Real-time PCR using Taqman Gene Expression Assays and the Applied Biosystems 7300 System (Applied Biosystems, Foster City, CA, USA) was used to compare steady-state mRNA levels. Taqman Gene Expression Assays contain two unlabeled primers specific to the gene of interest used at a final concentration of $900 \mathrm{nM}$ each, as well as a FAM dye-labeled, minor groove binder (MGB)-containing probe used at a final concentration of $250 \mathrm{nM}$. All assays have an amplification efficiency of $100 \%$ within measurement error $( \pm 10 \%)$. All assays used have been optimized for specificity, sensitivity, and efficiency by the manufacturer. Sequences of primers and alignment maps are referenced on the Applied Biosystems website. mRNA transcript levels of the following genes were assayed; gene symbols, followed by the Taqman Assay ID number of each primer/probe set, are in parentheses: Bmp15 (assay ID Mm00437797_m1), Cyp19a1 (Mm00484049_m1), DEAD (Asp-Glu-Ala-Asp) box polypeptide 4 (Ddx4; Mm00802445_m1), Figla (Mm00488691_m1), Fgf8
(Mm00438922_m1), FSH receptor (Fshr; Mm00442819_m1), Gdf9 (Mm00433565_m1), Igf1 (Mm00439561_m1), Inha (Mm00439683_m1), Inhba (Mm00434338_m1), Jag1 (Mm00496902_m1), Kit (Mm00445212_m1), Kitl (Mm00442972_m1), Lhcgr (Mm00442931_m1), NLR family, pyrin domain containing 5 (NIrp5; Mm00488691_m1), Nobox (Mm00453743_m1), Tgfbr2 (Mm00436977_m1), Zp1 (Mm00494367_m1), Zp2 (Mm00442173_m1), and Zp3 (Mm00442176_m1). Glyceraldehyde-3-phosphate dehydrogenase (Gapdh; 4352339E) was used as an endogenous control. In total, three independent samples were each run in triplicate, with in vivo samples isolated from different mice and in vitro samples isolated from different mice and cultured separately as described above. Reactions run without reverse transcriptase served as negative controls.

\section{Statistical analysis}

Follicle size, survival rate, antral growth rate, and gene expression data were collected for three independent cultures for each time point. Gene expression relative to 2 LS follicles was determined using the comparative $C_{\mathrm{t}}$ method. Statistical significance for gene expression was analyzed using ANOVA followed by Tukey post hoc test.

\section{Declaration of interest}

The authors declare that there is no conflict of interest that could be perceived as prejudicing the impartiality of the research reported.

\section{Funding}

This work has been funded by the NIH U54 HD041857 grant.

\section{References}

Albertini DF, Combelles CM, Benecchi E \& Carabatsos MJ 2001 Cellular basis for paracrine regulation of ovarian follicle development. Reproduction 121 647-653. (doi:10.1530/rep.0.1210647)

Bristol SK \& Woodruff TK 2004 Follicle-restricted compartmentalization of transforming growth factor beta superfamily ligands in the feline ovary. Biology of Reproduction 70 846-859. (doi:10.1095/biolreprod.103. 021857)

Cortvrindt R, Smitz J \& Van Steirteghem AC 1996 In-vitro maturation, fertilization and embryo development of immature oocytes from early preantral follicles from prepuberal mice in a simplified culture system. Human Reproduction 11 2656-2666.

Dawson E, Mapili G, Erickson K, Taqvi S \& Roy K 2008 Biomaterials for stem cell differentiation. Advanced Drug Delivery Reviews 60 215-228. (doi:10.1016/j.addr.2007.08.037)

Dean J 2002 Oocyte-specific genes regulate follicle formation, fertility and early mouse development. Journal of Reproductive Immunology $\mathbf{5 3}$ 171-180. (doi:10.1016/S0165-0378(01)00100-0)

Dong J, Albertini DF, Nishimori K, Kumar TR, Lu N \& Matzuk MM 1996 Growth differentiation factor-9 is required during early ovarian folliculogenesis. Nature 383 531-535. (doi:10.1038/383531a0)

Driancourt MA, Reynaud K, Cortvrindt R \& Smitz J 2000 Roles of kit and kit ligand in ovarian function. Reviews of Reproduction 5 143-152. (doi:10. 1530/ror.0.0050143)

Epifano O, Liang LF, Familari M, Moos MC Jr \& Dean J 1995 Coordinate expression of the three zona pellucida genes during mouse oogenesis. Development 121 1947-1956. 
Eppig JJ \& Schroeder AC 1989 Capacity of mouse oocytes from preantral follicles to undergo embryogenesis and development to live young after growth, maturation, and fertilization in vitro. Biology of Reproduction 41 268-276. (doi:10.1095/biolreprod41.2.268)

Eppig JJ, Wigglesworth K \& Pendola FL 2002 The mammalian oocyte orchestrates the rate of ovarian follicular development. PNAS 99 2890-2894. (doi:10.1073/pnas.052658699)

Erickson GF \& Shimasaki S 2000 The role of the oocyte in folliculogenesis. Trends in Endocrinology and Metabolism 11 193-198. (doi:10.1016/ S1043-2760(00)00249-6)

Erickson GF \& Shimasaki S 2001 The physiology of folliculogenesis: the role of novel growth factors. Fertility and Sterility 76 943-949. (doi:10.1016/ S0015-0282(01)02859-X)

Findlay JK, Drummond AE, Dyson M, Baillie AJ, Robertson DM \& Ethier JF 2001 Production and actions of inhibin and activin during folliculogenesis in the rat. Molecular and Cellular Endocrinology 180 139-144. (doi:10.1016/S0303-7207(01)00521-4)

Griffin J, Emery BR, Huang I, Peterson CM \& Carrell DT 2006 Comparative analysis of follicle morphology and oocyte diameter in four mammalian species (mouse, hamster, pig, and human). Journal of Experimental \& Clinical Assisted Reproduction 3 2. (doi:10.1186/1743-1050-3-2)

Hickey GJ, Chen SA, Besman MJ, Shively JE, Hall PF, Gaddy-Kurten D \& Richards JS 1988 Hormonal regulation, tissue distribution, and content of aromatase cytochrome P450 messenger ribonucleic acid and enzyme in rat ovarian follicles and corpora lutea: relationship to estradiol biosynthesis. Endocrinology 122 1426-1436. (doi:10.1210/endo-122-41426)

Juengel JL \& McNatty KP 2005 The role of proteins of the transforming growth factor-beta superfamily in the intraovarian regulation of follicular development. Human Reproduction Update 11 143-160. (doi:10.1093/ humupd/dmh061)

Knight PG \& Glister C 2006 TGF-beta superfamily members and ovarian follicle development. Reproduction 132 191-206. (doi:10.1530/rep.1. 01074)

Kreeger PK, Fernandes NN, Woodruff TK \& Shea LD 2005 Regulation of mouse follicle development by follicle-stimulating hormone in a threedimensional in vitro culture system is dependent on follicle stage and dose. Biology of Reproduction 73 942-950. (doi:10.1095/biolreprod. 105.042390)

Kreeger PK, Deck JW, Woodruff TK \& Shea LD 2006 The in vitro regulation of ovarian follicle development using alginate-extracellular matrix gels. Biomaterials 27 714-723. (doi:10.1016/j.biomaterials.2005.06.016)

LaPolt PS, Tilly JL, Aihara T, Nishimori K \& Hsueh AJ 1992 Gonadotropininduced up- and down-regulation of ovarian follicle-stimulating hormone $(\mathrm{FSH})$ receptor gene expression in immature rats: effects of pregnant mare's serum gonadotropin, human chorionic gonadotropin, and recombinant FSH. Endocrinology 130 1289-1295. (doi:10.1210/en. 130.3.1289)

Lei ZM, Mishra S, Zou W, Xu B, Foltz M, Li X \& Rao CV 2001 Targeted disruption of luteinizing hormone/human chorionic gonadotropin receptor gene. Molecular Endocrinology 15 184-200. (doi:10.1210/ me.15.1.184)

McGee EA \& Hsueh AJ 2000 Initial and cyclic recruitment of ovarian follicles. Endocrine Reviews 21 200-214. (doi:10.1210/er.21.2.200)

O'Brien MJ, Pendola JK \& Eppig JJ 2003 A revised protocol for in vitro development of mouse oocytes from primordial follicles dramatically improves their developmental competence. Biology of Reproduction 68 1682-1686. (doi:10.1095/biolreprod.102.013029)

Otsuka F, Yao Z, Lee T, Yamamoto S, Erickson GF \& Shimasaki S 2000 Bone morphogenetic protein-15. Identification of target cells and biological functions. Journal of Biological Chemistry 275 39523-39528. (doi:10. 1074/jbc.M007428200)
Pangas SA, Rademaker AW, Fishman DA \& Woodruff TK 2002 Localization of the activin signal transduction components in normal human ovarian follicles: implications for autocrine and paracrine signaling in the ovary. Journal of Clinical Endocrinology and Metabolism 87 2644-2657. (doi:10.1210/jc.87.6.2644)

Parrott JA \& Skinner MK 1997 Direct actions of kit-ligand on theca cell growth and differentiation during follicle development. Endocrinology 138 3819-3827. (doi:10.1210/en.138.9.3819)

Richards JS, Russell DL, Ochsner S, Hsieh M, Doyle KH, Falender AE, Lo YK \& Sharma SC 2002 Novel signaling pathways that control ovarian follicular development, ovulation, and luteinization. Recent Progress in Hormone Research 57 195-220. (doi:10.1210/rp.57.1.195)

Segaloff DL, Wang HY \& Richards JS 1990 Hormonal regulation of luteinizing hormone/chorionic gonadotropin receptor mRNA in rat ovarian cells during follicular development and luteinization. Molecular Endocrinology 4 1856-1865. (doi:10.1210/mend-4-12-1856)

Shimasaki S, Moore RK, Otsuka F \& Erickson GF 2004 The bone morphogenetic protein system in mammalian reproduction. Endocrine Reviews 25 72-101. (doi:10.1210/er.2003-0007)

Sites CK, Patterson K, Jamison CS, Degen SJ \& LaBarbera AR 1994 Folliclestimulating hormone (FSH) increases FSH receptor messenger ribonucleic acid while decreasing FSH binding in cultured porcine granulosa cells. Endocrinology 134 411-417. (doi:10.1210/en.134.1.411)

Suzumori N, Yan C, Matzuk MM \& Rajkovic A 2002 Nobox is a homeoboxencoding gene preferentially expressed in primordial and growing oocytes. Mechanisms of Development 111 137-141. (doi:10.1016/ S0925-4773(01)00620-7)

Thomas FH \& Vanderhyden BC 2006 Oocyte-granulosa cell interactions during mouse follicular development: regulation of kit ligand expression and its role in oocyte growth. Reproductive Biology and Endocrinology 4 19. (doi:10.1186/1477-7827-4-19)

Vorontchikhina MA, Zimmermann RC, Shawber CJ, Tang H \& Kitajewski J 2005 Unique patterns of Notch1, Notch4 and Jagged1 expression in ovarian vessels during folliculogenesis and corpus luteum formation. Gene Expression Patterns 5 701-709. (doi:10.1016/j.modgep.2005.02. 001)

West ER, Shea LD \& Woodruff TK 2007a Engineering the follicle microenvironment. Seminars in Reproductive Medicine 25 287-299. (doi:10.1055/s-2007-980222)

West ER, Xu M, Woodruff TK \& Shea LD 2007b Physical properties of alginate hydrogels and their effects on in vitro follicle development. Biomaterials 28 4439-4448. (doi:10.1016/j.biomaterials.2007.07.001)

Woodruff TK, D'Agostino J, Schwartz NB \& Mayo KE 1988 Dynamic changes in inhibin messenger RNAs in rat ovarian follicles during the reproductive cycle. Science 239 1296-1299. (doi:10.1126/science. 3125611)

Xu M, Kreeger PK, Shea LD \& Woodruff TK 2006a Tissue-engineered follicles produce live, fertile offspring. Tissue Engineering 12 2739-2746. (doi:10.1089/ten.2006.12.2739)

Xu M, West E, Shea LD \& Woodruff TK $2006 b$ Identification of a stagespecific permissive in vitro culture environment for follicle growth and oocyte development. Biology of Reproduction 75 916-923. (doi:10. 1095/biolreprod.106.054833)

Received 23 November 2010

First decision 14 January 2011

Revised manuscript received 10 May 2011

Accepted 24 May 2011 